\title{
Bone protection by estrens occurs through non-tissue-selective activation of the androgen receptor
}

\author{
Sara H. Windahl, ${ }^{1}$ René Galien, ${ }^{2}$ Riccardo Chiusaroli, ${ }^{1}$ Philippe Clément-Lacroix, ${ }^{2}$ \\ Frederic Morvan, ${ }^{1}$ Liên Lepescheux, ${ }^{2}$ François Nique, ${ }^{2}$ William C. Horne, ${ }^{1}$ \\ Michèle Resche-Rigon, ${ }^{2}$ and Roland Baron ${ }^{1,3}$
}

\begin{abstract}
1'Department of Orthopaedics, Yale University School of Medicine, New Haven, Connecticut, USA. 2ProStrakan Pharmaceuticals, Romainville, France. ${ }^{3}$ Department of Cell Biology, Yale University School of Medicine, New Haven, Connecticut, USA.
\end{abstract}

\begin{abstract}
The use of estrogens and androgens to prevent bone loss is limited by their unwanted side effects, especially in reproductive organs and breast. Selective estrogen receptor modulators (SERMs) partially avoid such unwanted effects, but their efficacy on bone is only moderate compared with that of estradiol or androgens. Estrens have been suggested to not only prevent bone loss but also exert anabolic effects on bone while avoiding unwanted effects on reproductive organs. In this study, we compared the effects of a SERM (PSK3471) and 2 estrens (estren- $\alpha$ and estren- $\beta$ ) on bone and reproductive organs to determine whether estrens are safe and act via the estrogen receptors and/or the androgen receptor (AR). Estrens and PSK3471 prevented gonadectomy-induced bone loss in male and female mice, but none showed true anabolic effects. Unlike SERMs, the estrens induced reproductive organ hypertrophy in both male and female mice and enhanced MCF-7 cell proliferation in vitro. Estrens directly activated transcription in several cell lines, albeit at much higher concentrations than estradiol or the SERM, and acted for the most part through the AR. We conclude that the estrens act mostly through the AR and, in mice, do not fulfill the preclinical efficacy or safety criteria required for the treatment or prevention of osteoporosis.
\end{abstract}

\section{Introduction}

Bone mass is maintained at least in part by estrogens and androgens acting on their receptors (ERs and AR, respectively) in target cells. Estrogen or androgen deficiency is associated with a rapid decrease in bone mass that often develops into osteoporosis. Although estrogens and androgens are known to prevent age-, gonadectomy-, or menopause-induced bone loss, their use is limited by their lack of specificity, resulting in unwanted side effects in other targets organs and in particular reproductive organs and breast (1). Such unwanted effects have been at least partially avoided through the development of selective estrogen receptor modulators (SERMs) that display tissue selectivity, being neutral or even behaving as anti-estrogens on breast and uterus, while being estrogenic and preventing bone resorption in bone (2-4).

The first generation of SERMs, exemplified by tamoxifen, already offered significant improvement compared with estradiol $\left(\mathrm{E}_{2}\right)$ in the treatment of bone loss in postmenopausal women, while preventing breast cancer (5). However, chronic administration of tamoxifen has been suggested to be associated with increased risk of uterine cancer $(6,7)$. Raloxifene, a second-generation SERM, and the first SERM that received FDA approval for prevention

\footnotetext{
Nonstandard abbreviations used: AR, androgen receptor; ARE, androgen response element; DHT, $5 \alpha$-dihydrotestosterone; Dpyr, deoxypyridinoline; $\mathrm{E}_{2}$, estradiol; ER, estrogen receptor; ERE, estrogen response element; ORX, orchidectomized, orchidectomy; OVX, ovariectomized, ovariectomy; PR, progesterone receptor; SARM, selective androgen receptor modulator; SERM, selective estrogen receptor modulator. Conflict of interest: R. Galien, P. Clément-Lacroix, L. Lepescheux, F. Nique, M. Resche-Rigon, and R. Baron are currently or have been employed by ProStrakan Pharmaceuticals (previously ProSkelia Pharmaceuticals) and also own stock options in that company.

Citation for this article: J. Clin. Invest. 116:2500-2509 (2006). doi:10.1172/JCI28809.
}

and treatment of postmenopausal osteoporosis, has been shown to avoid breast and uterine responses and thus to be safer (8).

So far, all described SERMs are effective at partially preventing bone loss, albeit not as effective as estrogens or androgens. This effect is mediated mostly through inhibition of bone resorption. In contrast to parathyroid hormone, and to a certain extent to estrogens and androgens themselves, these compounds are not able to anabolically activate bone formation and restore bone mass. This has fueled the search for other ligands that could improve upon existing SERMs, maintaining their selectivity and safety while increasing their ability to prevent or treat bone loss via a more potent prevention of bone resorption and/or a true anabolic effect on osteoblasts. Estrens have recently been suggested to constitute such a new class of compounds, capable not only of antiresorptive action but also of anabolic effects on bone $(9,10)$. Moreover, these compounds are reported to affect bone in both males and females, as they act through both the ERs and the AR, while also failing to promote the hypertrophy of, and thus being safe for, reproductive organs $(10,11)$. The cellular effects of estrens have been attributed to activation of membrane signaling through the MAPK cascade and not to direct activation of gene regulation, in contrast to estrogens, androgens, or SERMs. Hence the use of the term ANGELS (activators of non-genomic estrogen-like signaling) to describe this family of compounds $(12,13)$.

Because no study has directly compared SERMs and estrens in animal models, it has not been possible to determine whether these compounds truly offer a significant advantage over SERMs. There is great therapeutic potential for compounds that would not only prevent bone loss but also restore bone mass through anabolic action on bone formation, while being selective and 


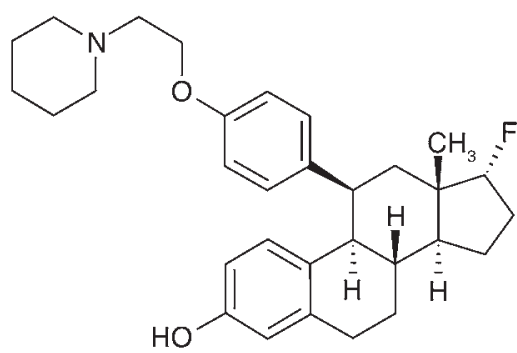

Figure 1

Chemical structure of the SERM compound PSK3471.

avoiding the unwanted effects on reproductive organs and breast. The purpose of this study was thus to directly compare the effects of 2 estrens (4-estren-3 $\alpha, 17 \beta$-diol and its isomer 4-estren-3 $3,17 \beta$ diol; estren- $\alpha$ and estren- $\beta$, respectively) with those of a SERM currently in development (PSK3471; Figure 1), both on bone and on other target organs, in both females and males. Furthermore, we investigated the relative roles of the ERs and the AR in these responses through the use of genetically or pharmacologically altered mice to determine whether, as reported $(10,11)$, these compounds are gender neutral and could therefore be used in both males and females to restore bone mass. We show here that both estrens and PSK3471 were able to prevent gonadectomyinduced bone loss but that estrens did not display any anabolic effects on bone. In addition, estrens, but not PSK3471, induced reproductive organ hypertrophy and stimulated breast cancer cell proliferation. The effects on bone and reproductive organs were mainly mediated through the AR, with a possible contribution by ER $\alpha$. Estren- $\beta$ mimicked estren- $\alpha$ in all assays. We conclude that estren- $\alpha$ and estren- $\beta$ act mostly though the AR and, based on these animal studies, do not fulfill the criteria required of safe and selective modulators of sex hormone receptors for the treatment or prevention of osteoporosis.

\section{Results}

Estrens, delivered by subcutaneous slow-release pellets, have been suggested to be anabolic to bone and safe for reproductive organs. We determined their activity on bone and safety in reproductive organs in ovariectomized (OVX) female C57BL/ 6 mice, comparing their effects with those of $\mathrm{E}_{2}$ and a SERM (PSK3471). As shown in Figure 2, estren- $\alpha$ partially prevented bone loss after OVX but did so less efficiently than $\mathrm{E}_{2}$ or PSK3471 at the tested doses. Histomorphometric analysis and biochemical indices of bone turnover showed that, under these experimental conditions - where all compounds are delivered by subcutaneous pellets, ensuring a constant and sustained release over the entire experimental period $-\mathrm{E}_{2}$ and PSK3471 not only protected against OVX-induced bone loss but also induced an increase in bone volume compared with sham treatment. This occurred despite a surprising increase in the number of osteoclasts, possibly linked to the unusual delivery mode used here in order to allow comparison with estren- $\alpha$. It should be noted, however, that the activity of these cells was decreased overall, as shown by deoxypyridinoline (Dpyr) measurements and the increase in bone volume. Estren- $\alpha$ reduced the otherwise increased postOVX bone turnover more efficiently than either $\mathrm{E}_{2}$ or PSK3471 (Figure 3 ) but also reduced the bone formation rate significantly, in contrast to the other treatments. These results therefore suggest that estren- $\alpha$ is not a true bone anabolic agent but rather an agent that controls bone turnover, with a mildly positive overall balance at the dose tested here. When the safety features of this compound were tested in the OVX female mice, we found that estren- $\alpha$ increased the overall cross-sectional area of both the myometrium and the endometrium in the uterus at least as much as $E_{2}$ (Figure 4). In contrast, PSK3471 had only moderate, albeit still significant, effects on these 2 safety parameters. Furthermore, again like $\mathrm{E}_{2}$, estren- $\alpha$ stimulated the proliferation of MCF-7 cells in vitro (Figure 5), in sharp contrast to PSK3471, which, as expected for a SERM, acted as an antagonist in this cell system. Thus, according to these results, obtained with the same dose used in another study (10), estren- $\alpha$ is neither anabolic on bone nor inactive with regard to the uterus or to the proliferation of a human breast cancer cell line.

Three additional studies were then performed. First, we compared the effect of estren- $\alpha$ on the uterus in 2 separate mouse strains. As shown in Supplemental Figure 1 (supplemental material available online with this article; doi:10.1172/JCI28809DS1), uterus weight decreased, as expected, after OVX but was unexpectedly and significantly increased beyond control values by estren- $\alpha$ in both C57BL/ 6 and Swiss Webster mice. These results exclude the possibility that the stimulation of female reproductive organs by estren- $\alpha$ could be due to different responses in various strains of mice. Second, we compared the ability of estrens and PSK3471 to bind to the ERs, AR, and other steroid receptors, measuring their affinities relative to those of each receptor's natural ligand. As shown in Table 1, estren- $\alpha$ exhibited 10- to 15-fold higher relative affinity for the AR than for ER $\alpha$ and $E R \beta$, in sharp contrast to PSK3471, whose relative affinities for the 2 estrogen receptors $\mathrm{ER} \alpha$ and $\mathrm{ER} \beta$ were 5- to 6 -fold higher than its relative affinity for the AR. Neither compounds had significant binding affinity for other steroid receptors, although estrens, but not PSK3471, activated the progesterone receptor (PR) in a transcriptional assay (see below). These results therefore indicated, unexpectedly, that estren- $\alpha$ may exert some or all of its effects, whether on bone or

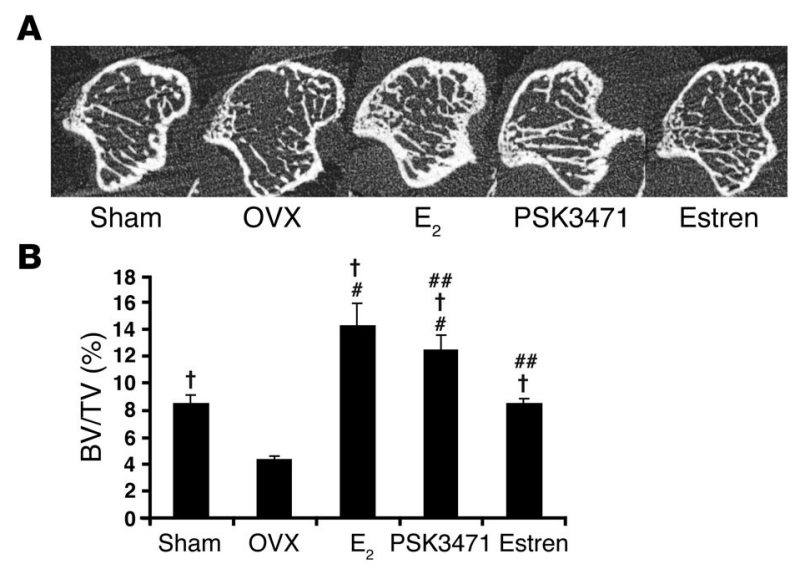

Figure 2

Estren- $\alpha$ prevents bone loss in OVX female mice. (A) Representative $\mu C T$ sections of tibiae from sham-operated or OVX mice treated with implanted slow-release pellets delivering $E_{2}$, PSK3471, or estren- $\alpha$. (B) Quantification of $\mu \mathrm{CT}$ analysis. Values are mean \pm SEM. ${ }^{\dagger} P<0.001$ versus OVX mice; $P<0.01$ versus sham-operated mice; \# $P<0.01$ versus $\mathrm{E}_{2}$-treated mice. $\mathrm{BV} / \mathrm{TV}$, bone volume/tissue volume. 

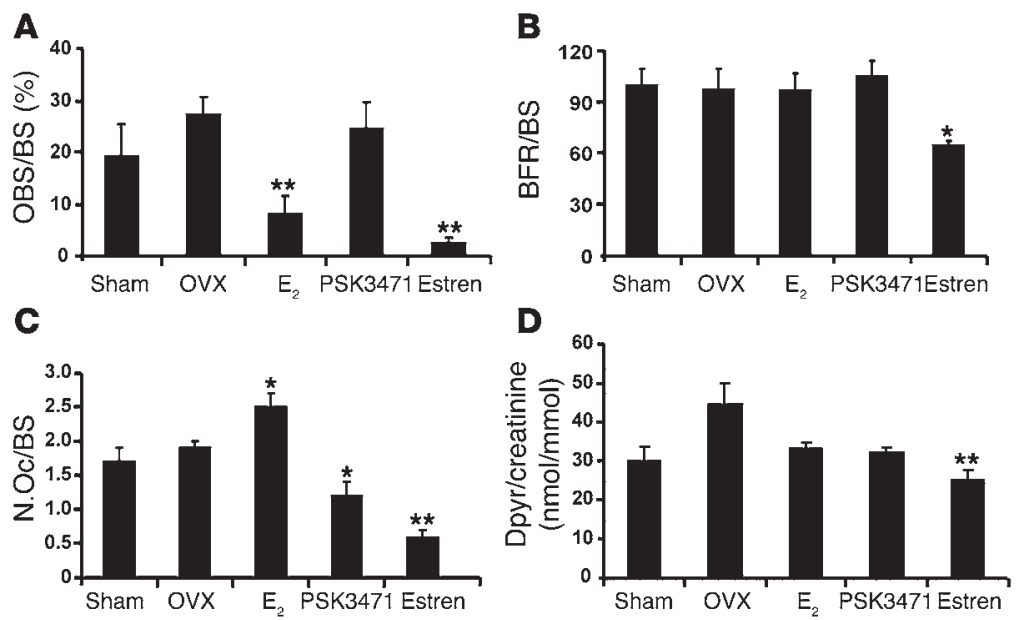

Figure 3

Estren- $\alpha$ reduces bone resorption and bone formation in OVX female mice. Histomorphometric analysis was performed on tibiae, and urinary Dpyr concentration, a biochemical index of bone resorption, was measured in urine. All compounds were delivered by subcutaneous slow-release pellets. In contrast to $E_{2}$ and PSK3471 treatment, estren- $\alpha$ treatment decreased bone formation compared with OVX alone, as shown by the decreased osteoblast surface (OBS) as a percentage of trabecular bone surface (BS) (A) and the bone formation rate (BFR/BS, $\mu \mathrm{m}^{3} / \mu \mathrm{m}^{2} / \mathrm{yr}$ ) (B). PSK3471 did not modify the bone formation; osteoblast surface and bone formation rate remained high in treated animals and were not significantly altered compared with those in OVX mice. Estren- $\alpha$ treatment also decreased the bone resorption compared with that in OVX mice as shown by the lowered osteoclast number (N.Oc/BS) (C) and urinary Dpyr cross-links (D). PSK3471 also decreased the osteoclast numbers compared with OVX alone, whereas under these experimental conditions, $E_{2}$ reduced Dpyr but not the number of osteoclasts. ${ }^{\star} P<0.05$ and ${ }^{\star \star} P<0.01$ versus OVX mice.

on reproductive organs, via the AR, consistent with the recently reported results of 2 other studies $(14,15)$.

We have also determined the relative abilities of estren- $\alpha$ to activate transcription through the ER and $\mathrm{AR}$, compared with those of $\mathrm{E}_{2}$ and $5 \alpha$-dihydrotestosterone (DHT), respectively. As shown in Supplemental Figure 2, estren- $\alpha$ was a very weak activator of ER $\alpha$ and ER $\beta$, with about 3 orders of magnitude less activity than $E_{2}$. In contrast, estren- $\alpha$ strongly activated the AR, with only a 1 order of magnitude difference relative to DHT. These results suggest that, provided that a high enough concentration of the compound reaches target organs, estren- $\alpha$ can activate both ER- and AR-dependent transcription, although the $\mathrm{AR}$ is much more likely to be activated than the ER at the doses used in already-published studies and here.

To test this hypothesis and distinguish the ER-mediated and the AR-mediated effects of estren- $\alpha$ on the reproductive organs, we combined estren- $\alpha$ treatment with pure anti-estrogen (PSK3668) or pure anti-androgen (RU58642) compounds. As shown in Figure 6, both the anti-estrogen and the anti-androgen inhibited the estren- $\alpha$-induced increase in uterine weight in WT mice, but the anti-androgen was significantly more efficient than the anti-estrogen. These results establish the fact that estren- $\alpha$ exerts its effect on female reproductive organs predominantly via the AR, albeit in part via the ER.

We concluded from these studies in WT female mice that estren- $\alpha$ is capable of strongly decreasing bone turnover after OVX, yet it did not show any anabolic effects and, at the dose tested here, was less efficient than $\mathrm{E}_{2}$ or PSK3471 at protecting bone mass. Furthermore, estren- $\alpha$ negatively affected both the uterus in vivo and the proliferation of human breast cancer cells in vitro. Estren- $\alpha$ bound more efficiently to the AR than to the ERs, and most of its negative impact on the uterus was mediated via the AR. Estren- $\alpha$ therefore appeared, in females, as a mixed androgen-estrogen, with no dissociation of effects on bone and reproductive organs.

Given the dominant androgen-like features of estren- $\alpha$, and in order to further explore the effects of the estrens on reproductive organs and the role of ERs in the observed response, we then performed a series of studies in male mice. As shown in Figure 7, estren- $\alpha$ was capable of preventing bone loss after orchidectomy (ORX) and did so as efficiently as DHT and PSK3471. This contrasted with its relatively weak effects in female mice (Figure 2). Detailed histomorphometry demonstrated that estren- $\alpha$ was as efficient as DHT at reducing ORX-induced bone turnover, including a marked decrease in bone formation (Figure 8). In contrast, PSK3471 had little or no effect on measured parameters of bone turnover, notwithstanding the fact that it protected from bone loss in males, suggesting that it induces a positive balance between bone formation and resorption.

To determine the role of the ER in these responses, we then used $\mathrm{ER}^{-/-}$male mice, in which, as we have previously shown (16), ER $\beta$ does not contribute in any measurable way to bone responses. In these mice, estren- $\alpha$ was as efficient as DHT in protecting bone mass and restoring bone turnover, and the entire protective effect was lost in the presence of anti-androgen (Figure 9). These results firmly established both that estren- $\alpha$ exerts most of its effects on bone via the AR and that, at least in males, the ER seems to play no role in the observed responses.

We then asked whether estren- $\alpha$ was inducing a hypertrophic effect on the male reproductive organs and, if so, what the role of the ERs versus AR, was. As shown in Figure 10, estren- $\alpha$ was as potent as DHT at inducing an increase in the weight of seminal vesicles, whereas PSK3471 had no effect. This increase in seminal vesicle weight was entirely blocked by anti-androgens and was not altered in $\mathrm{ER}^{-/-}$mice (Figure 10C). Thus, these results establish firmly the fact that while estren- $\alpha$ is very efficient at protecting against bone loss and preventing the increased bone turnover after ORX in male mice, it behaves as a pure androgen, acting only via the AR, like DHT, and fails to selectively affect bone and not the reproductive tract.

To more extensively explore this last point, we then determined whether the dose response of bone and male reproductive organs could be different, offering a potential safety window within which bone could be protected without affecting seminal vesicles. As shown in Supplemental Figure 3, progressively decreasing the administered dose of estren- $\alpha$ in WT mice led to a progressive loss of the bone-protective effects at the same dose as effects on seminal vesicle began to be lost. Thus, it appears that estren- $\alpha$ is not a selective AR modulator.

Finally, we tested the estren- $\beta$ isomer in all the experiments described above in order to determine whether its properties would differ from those of estren- $\alpha$. Estren- $\beta$ exhibited essentially the same effects as estren- $\alpha$ in all the tests (data not shown). 
A

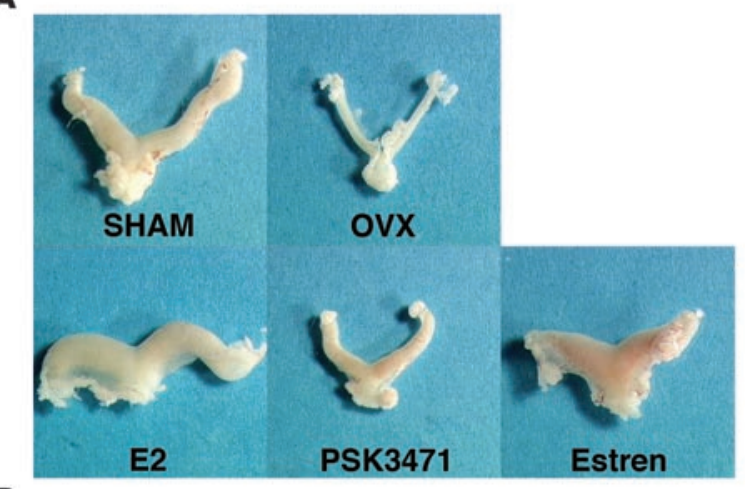

B

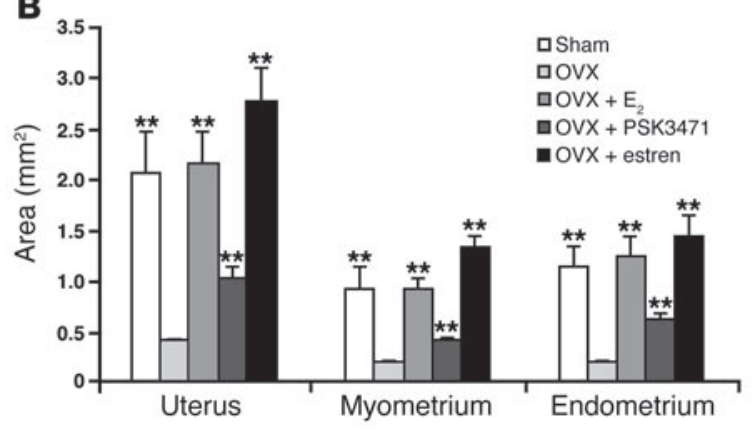

Namely, it prevented gonadectomy-induced bone loss; elicited a hypertrophic response in reproductive organs; bound to both ERs and with higher affinity to the AR; activated AR-dependent transcription more efficiently than ER-dependent transcription; was unable to antagonize $\mathrm{E}_{2}$-induced proliferation of MCF-7 cells; and acted mostly via the AR in vivo.

\section{Discussion}

As well illustrated by the results of the Women's Health Initiative and the Million Women studies (17-19), the medical need for compounds that selectively retain the benefits of estrogens on bone while avoiding their possibly detrimental effects on other target organs, in particular the breast and uterus, remains very high. Similarly, and despite the fact that androgens have beneficial effects on bone and muscle mass, their negative impact on the prostate prevents their widespread use in the treatment of osteoporosis and frailty in males (20). This dual and important medical need has led to the development of several SERMs and the marketing of some $(1,5,8)$ as well as the search for selective androgen receptor modulators (SARMs), some of which are currently in preclinical or clinical development $(21,22)$.

In a recent and very provocative series of articles, estren- $\alpha$, an $E_{2}$-like compound, has been reported to fulfill not only the efficacy criteria of a SERM on bone but also to maintain its safety with respect to breast and uterus, as well as those of a SARM with respect to prostate, thereby suggesting the possible therapeutic use of estren- $\alpha$ in both males and females (10).

\section{Figure 4}

Estren- $\alpha$ has deleterious effects on the uterus in WT mice. (A) Representative uteri from the mice in Figure 2. (B) Histomorphometric quantification of total uterus, myometrium, and endometrium areas. Estren- $\alpha$ was as uterotrophic as $E_{2}$, while PSK3471 induced only very moderate uterotrophic effects. ${ }^{\star \star} P<0.01$ versus OVX mice.

Furthermore, this compound was suggested to be a true bone anabolic agent (10) and to act on bone cells via a nongenotropic and gender-neutral signaling pathway $(10,11)$. Given the potential enormous importance of such a series of chemicals on the treatment of osteoporosis, the present study was undertaken to (a) compare directly the effects of a SERM with those of estren- $\alpha$ on bone; (b) further determine the safety margins of this compound by comparing several doses and their effects not only on bone but also on breast, the uterus, and the male reproductive organs; (c) determine whether the ERs, the AR, or both were required for the observed responses to estren treatment; and (d) further determine whether these compounds can achieve genotropic responses by the ER and/or AR at doses comparable to those used in in vivo experiments. In addition, this study determined whether estren- $\beta$, another member of the estren family of compounds, was also capable of inducing such effects.

Our results clearly demonstrate that although both estren- $\alpha$ and estren- $\beta$ are capable of preventing gonadectomy-induced bone loss in male and female mice, their effects on bone fail to be stronger than those of $\mathrm{E}_{2}$, DHT, or a SERM. Indeed, estrens were weaker than $E_{2}$ and the SERM in females and weaker than DHT but stronger than the SERM in males. Furthermore, no anabolic response, as would be measured by an increase in bone formation, was detected in treated animals. Only an $\mathrm{E}_{2}-$ or DHT-like repression of gonadectomy-induced high turnover could be detected following estren treatment. In terms of safety, we found that both estrens induced responses in reproductive organs in both males and females. While the difference in the ages of mice used in our study ( 3 months old) and the first study (6 and 8 months old) (10) might explain the difference in the

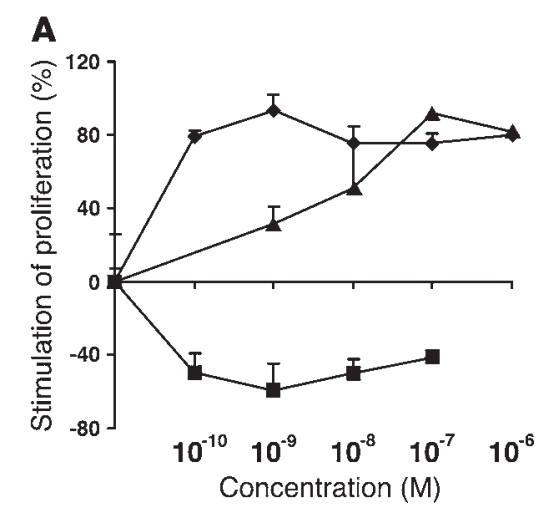

Figure 5

Estren- $\alpha$, but not PSK3471, stimulates breast cancer cell proliferation. (A) Estren- $\alpha$ significantly enhanced cellular proliferation in MCF-7 cells. Indeed, at $10 \mathrm{nM}$, estren- $\alpha$ and $\mathrm{E}_{2}$ displayed the same efficacy. PSK3471, in contrast, did not display any agonist activity on MCF-7 proliferation. (B) The experiment was performed as in A, but the ability of each compound to antagonize $\mathrm{E}_{2}$-induced cell proliferation was tested by cotreating the cells with $\mathrm{E}_{2}(0.1 \mathrm{nM})$ and increasing doses of compounds, as indicated. PSK3471, but not estren- $\alpha$, antagonized $E_{2}$-induced proliferation of MCF-7 cells. $E_{2}$, filled diamonds; estren- $\alpha$, filled triangles; PSK3471, filled squares. 


\section{Table 1}

Relative binding affinities of estrens and PSK3471 to ERs, ARs, and other steroid receptors

\begin{tabular}{lcccccccc}
\hline & \multicolumn{3}{c}{$\begin{array}{c}\text { Expressed in } \\
\text { COS-7 cells }\end{array}$} & \multicolumn{4}{c}{$\begin{array}{c}\text { Expressed in baculovirus- } \\
\text { infected Sf9 cells }\end{array}$} \\
& ER $\alpha$ & ER $\beta$ & AR & AR & PR & GR & MR \\
Estren- $\alpha$ & $0.15(2)$ & $0.2(2)$ & 2.5 & $4(3)$ & 0.7 & $<0.4$ & 0.15 \\
Estren- $\beta$ & $2.5(2)$ & 1.5 & 12.5 & $18(3)$ & 5.5 & 0.05 & 2.5 \\
PSK3471 & 6 & 5 & - & 1 & 1.5 & 0.8 & $<0.1$
\end{tabular}

The binding affinities of estren- $\alpha$, estren- $\beta$, and PSK3471 to recombinant human estrogen receptors (ER $\alpha, E R \beta), A R, P R$, glucocorticoid receptor (GR), and mineralocorticoid receptor (MR) were determined as described in Methods. The binding affinities of $E_{2}$ to the ERs, of testosterone to the AR, of dexamethasone to the GR, of progesterone to the PR, or of aldosterone to the MR were taken as reference (100\%). The numbers in parentheses indicate the number of experiments performed.

results - since it is known that the uterine response to estrogens decreases with age - Moverare et al. (23) also reported uterotrophic effects of estren- $\alpha$ in 11-month-old mice. Taken together, these results suggest strongly that estren- $\alpha$ induces uterotrophic responses at the doses used in these studies. This is in contrast with the SERM, which had been specifically selected for its lack of effects on the breast and uterus and whose safety in both female and male mice was confirmed in the present study. Surprisingly, both estrens also increased the proliferation of MCF-7 human breast cancer cells in vitro, whereas the SERM behaved as an antagonist to the proliferative effects of $E_{2}$, as expected. Finally, in males, both estrens induced unwanted responses in the seminal vesicles, comparable here again to DHT.

All the observations summarized above have purposefully been made at the dose of estren that was used in the initial reports on this compound (10). Given the discrepancy with this previous report, we also studied whether decreasing the dose of estrens or using the same strain of mice could lead to a better dissociation of the estrens' effects on reproductive organs and bone. As shown in Supplemental Figure 3, the effects on reproductive organs decreased at the same dose as the effects on bone started to decrease, suggesting that, independent of the dose, estren- $\alpha$ is not a selective modulator of the ER or AR. Finally, since the published reports used a Swiss Webster mouse strain, whereas our results were observed in C57BL/6 mice, we then repeated some of the experiments with Swiss Webster mice and again found no dissociation of the effects on bone and reproductive tissues or anabolic responses in mice treated with estren- $\alpha$.

In terms of mechanism of action, and in agreement with several recent reports $(11,15,24)$, we found that estren- $\alpha$ binds only weakly to ERs and more strongly to AR. In addition, we found that estren- $\beta$ also binds to the ERs and AR and that both compounds also bind to PR. More importantly, estren- $\alpha$ and estren- $\beta$, but not the SERM tested here, exhibited strong progestin agonist activity (data not shown) in HeLa cells transfected with a progesterone response element-containing promoter, indicating that these compounds are relatively nonselective steroids. Both estren- $\alpha$ and estren- $\beta$ induced transcriptional activity on different synthetic or natural estrogen response element-containing (EREcontaining) promoters via both $\mathrm{ER} \alpha$ and $\mathrm{ER} \beta$, and in different cell contexts where only the AF2 (HeLa cells) or both AF1 and $\mathrm{AF} 2$ domains of ER $\alpha$ (MDA cells) are required to fully stimulate transcription. Similarly, and in agreement with other reports (15, $23,24)$, we found that estren- $\alpha$ and estren- $\beta$ bind to the AR and stimulate transactivation of androgen response element-containing (ARE-containing) promoters with an efficacy much closer to that of DHT (1 log difference) than for ERE relative to $\mathrm{E}_{2}$ (3 log difference). This suggests that estrens are mostly behaving as androgens rather than estrogens.

The androgen-like behavior of estrens was further confirmed, both in males and in females, by the use of anti-androgens and anti-estrogens as well as ER $\alpha$-knockout mice. As shown in Figure 6, the addition of the anti-estrogen reduced the response of the uterus to estren by less than half, whereas the addition of the anti-androgen almost completely blocked the response to estren. The effect of estren on seminal vesicles in male ER $\alpha$-knockout mice was also completely blocked by the anti-androgen. Interestingly, our results demonstrate, in agreement with a recent report (25), that an androgen-like compound that cannot be aromatized, such as estren- $\alpha$, is also able to elicit uterine responses acting via the AR. It will be of major interest for the development of SARMs for application in female osteoporosis to determine whether this also applies to women.

More recently, Centrella et al. reported that estren- $\alpha$ is rapidly converted in osteoblasts into 19-nortestosterone, a potent anabolic steroid, by $3 \alpha$-hydroxysteroid dehydrogenase (14). This conversion also occurs in HeLa cells exposed to estren- $\alpha$ and to estren- $\beta$ as well, albeit to a lesser extent (data not shown). We therefore cannot exclude the possibility that the androgenic activity of estren- $\alpha$ and estren- $\beta$ detected in our HeLa cells might be partly due to 19-nortestosterone production, nor can we exclude the possibility that the proliferative effects of estren- $\alpha$ and estren$\beta$ on MCF-7 cells are not due to the progestative effects of 19 -nortestosterone, although estren- $\beta$ affected MCF-7 cell proliferation to a larger extent than did estren- $\alpha$, which is converted to 19 -nortestosterone more efficiently than estren- $\beta$. In addition, it is also possible that estren- $\alpha$ has androgenic effects on seminal vesicles because of its conversion to 19-nortestosterone.

We show in this study that similar doses of estren- $\alpha$ prevent bone loss and induce hypertrophy of seminal vesicles in both WT and ER $\alpha^{-/-}$ORX male mice. Since we previously established

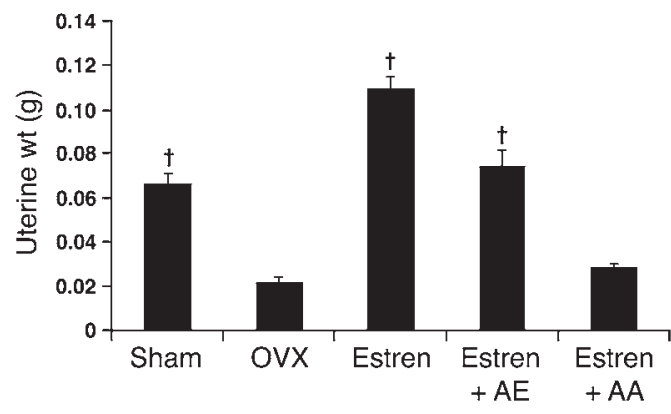

\section{Figure 6}

Estren- $\alpha$ effects on uterus are mediated by ERs and AR. WT C57BL/6 mice were subjected to sham operation, OVX, or OVX and treatment with estren- $\alpha$ pellets with or without subcutaneous coadministration of the anti-estrogen RU58668 (AE) or the anti-androgen RU58642 (AA). Cotreatment with anti-estrogen decreased the uterotrophic effects of estren by approximately $30 \%$, while cotreatment with the anti-androgen nearly abolished the uterotrophic effects of estren- $\alpha .{ }^{\dagger} P<0.001$ versus untreated OVX mice. 
A

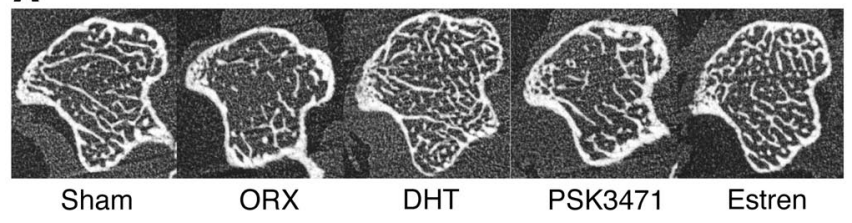

B

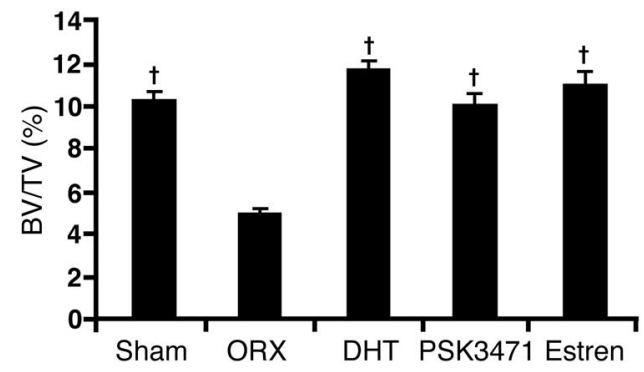

that ER $\beta$ was totally dispensable in males (16), this observation suggests that estren- $\alpha$ signaling in vivo is most likely exclusively mediated via AR in males. Again we cannot exclude that the in vivo androgenic activity of estren- $\alpha$ is not an effect resulting from its transformation into 19-nortestosterone (14), although a recent study by Hewitt et al. suggested that the estren conversion to 19-nortestosterone is not complete in vivo and that the uterine responses they saw after 24 hours were due to remaining estren or other metabolites (25). In vivo signaling of estren- $\alpha$ in females is not fully elucidated. Moverare et al. (23) hypothesized that estren$\alpha$ signals exclusively via ERs in female mice, since prevention of OVX-induced bone loss was lost in ER $\alpha^{-/-E R} \beta^{-/-}$mice. In contrast, a recent report showed that inhibition of the estren- $\alpha$ response in the mouse uterus required both an anti-estrogen and an anti-androgen. The estren effects were possibly induced by both nuclear ER $\alpha$ - and AR-mediated responses (25). Our study of uteri of female WT mice treated with estren- $\alpha$ with or without cotreatment with an antiestrogen or anti-androgen shows that the AR might play a larger role in estren- $\alpha$ uterine action than the ERs.

We found that the concentration of estren, whether acting through the ER, the $\mathrm{AR}$, or both, required to activate transcription was 100 - to 1,000 -fold higher than the required $E_{2}$ concentration in vitro but only 10 - to 100 -fold higher than the required DHT concentration. This will also favor AR-mediated responses upon estren treatment. It is, however, still unknown how that translates into the in vivo situation. In the in vivo studies described here (with the exception of the comparative study between the 2 mouse strains), we used 300-fold higher estren than $E_{2}$ concentrations, and, not knowing the pharmacokinetics and specific tissue concentrations of the estrens in mice, we cannot exclude the possibility that the

\section{Figure 8}

\section{Figure 7}

Estren- $\alpha$ prevents bone loss in ORX WT male mice. DHT, PSK3471, and estren- $\alpha$ are all fully protective against ORX-induced bone loss. (A) Representative $\mu \mathrm{CT}$ sections of tibiae from sham-operated or ORX mice treated with implanted slow-release pellets delivering DHT, PSK3471, or estren- $\alpha$. (B) The histomorphometric quantifications of $\mu \mathrm{CT}$ data. Values are mean $\pm \mathrm{SEM} ;{ }^{\dagger} P<0.001$ versus $\mathrm{ORX}$ mice.

effects we are seeing are due to direct transcriptional regulation by the AR and/or ER. It should be noted, however, that the same comment applies to the study by Kousteni et al. (10), and therefore in our view it is not possible to determine whether the mechanism of action of estrens in vivo is genotropic or nongenotropic.

Regarding safety in breast cancer cells, estren- $\alpha$ and estren- $\beta$ displayed significant agonist activity in HeLa cells stably expressing either ER $\alpha$ or ER $\beta$ and a synthetic ERE-glob-Luc reporter, in contrast to SERMs. Estrens also failed to show dose-dependent inhibition of $\mathrm{E}_{2}$-stimulated transcription, a characteristic antagonist activity of SERMs. In addition, we also found that estren- $\alpha$ weakly and estren- $\beta$ more efficiently stimulates transcription from the C3-Luc reporter in HeLa and MDA cells (data not shown), whereas PSK3471, raloxifene, and tamoxifen were totally inactive in these assays. Finally estren- $\alpha$ and estren- $\beta$ were compared with SERMs in MDA cells transiently transfected with a PS2-Luc reporter. Estren- $\alpha$ and tamoxifen displayed weak agonist activity, and estren- $\beta$ behaved as a full agonist, whereas PSK3471 and raloxifene again did not display any agonist activity (data not shown). Together these data indicate that estren- $\alpha$ and estren- $\beta$ not only elicit genomic responses at high concentrations when bound to either ER $\alpha$ or ER $\beta$ but also stimulate both AF1- and AF2-dependent responses. Furthermore, we found that estren- $\alpha$ and estren- $\beta$ stimulate MCF-7 cell prolif-
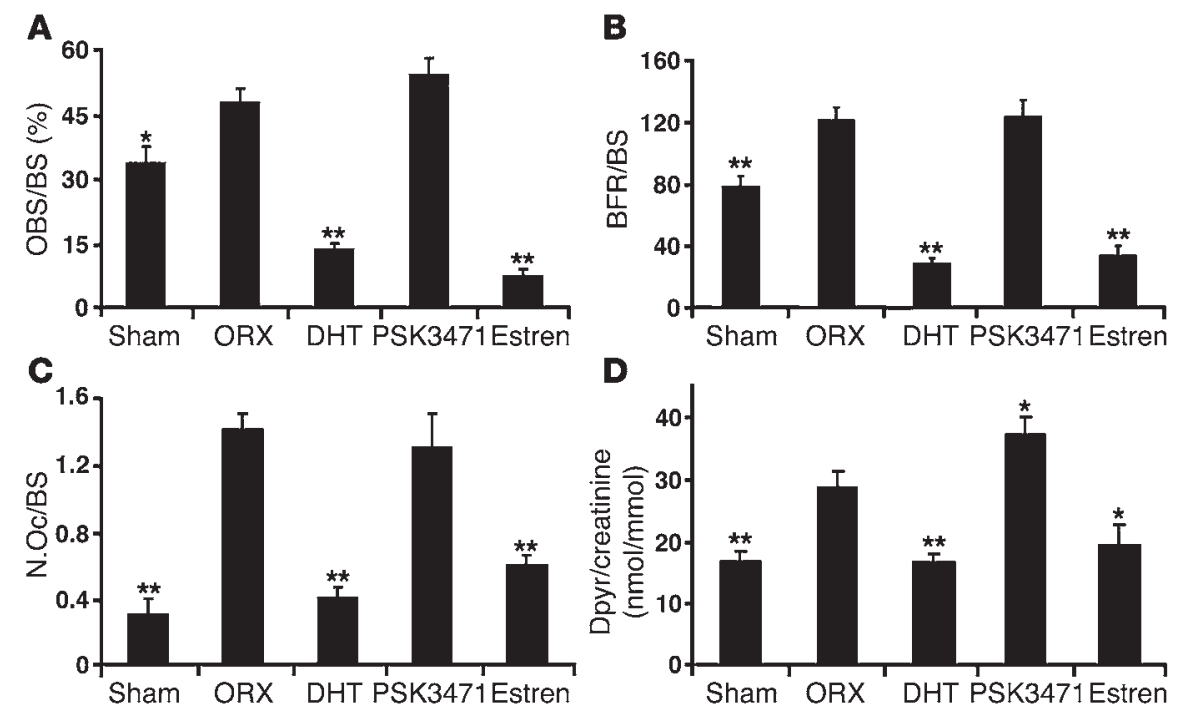

D

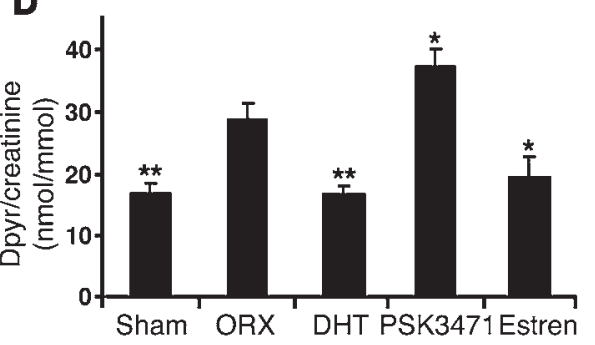

Estren- $\alpha$ reduces both bone resorption and bone formation in ORX male mice. Histomorphometric analysis was performed on tibiae from the mice described in Figure 7. Both estren- $\alpha$ and DHT reduced the bone formation parameters osteoblast surface (OBS/BS) (A) and bone formation rate $(\mathbf{B})$ and the bone resorption parameters osteoclast number $(\mathbf{C})$ and urinary Dpyr cross-links (D) in ORX male mice to levels at or below the levels seen in sham-operated animals. In contrast, PSK3471 had little effect on the ORX-induced increases in bone formation and bone resorption. ${ }^{\star} P<0.05$ and ${ }^{* *} P<0.01$ versus ORX mice. 

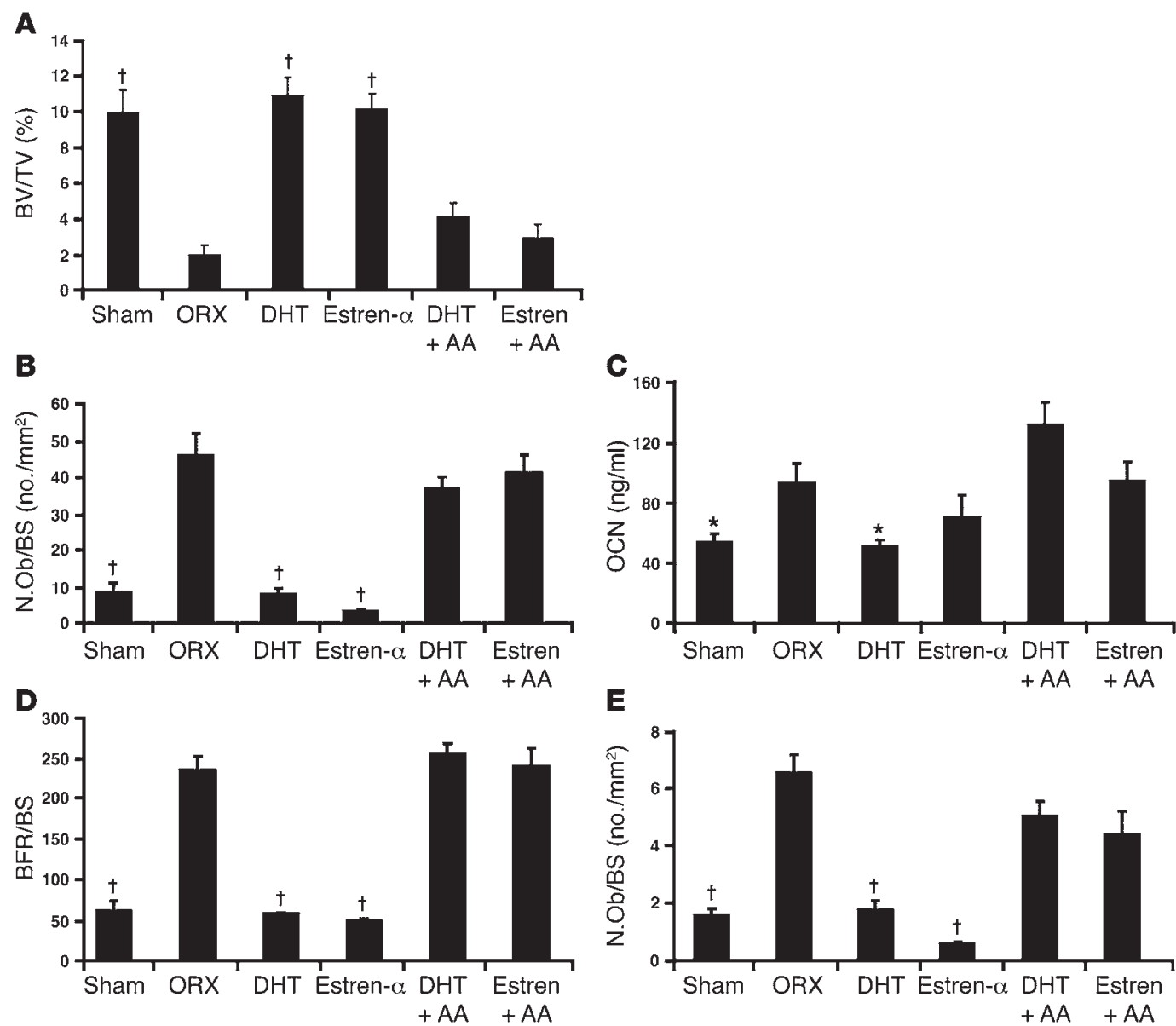

Figure 9

Estren- $\alpha$ protects bone in ORX ER $\alpha^{-/-}$male mice by restoring bone turnover. ER $\alpha^{-/}$mice were subjected to sham operation, ORX, or ORX and treatment with DHT or estren- $\alpha$, each with or without coadministration of the anti-androgen RU58642. DHT and estren prevented the ORXinduced decreases in cancellous bone volume (A) and increases in osteoblast number (N.Ob/BS) (B), serum osteocalcin (OCN) (C), bone formation rate (D), and osteoclast number (E). The effects of both DHT and estren- $\alpha$ were abolished by the simultaneous delivery of the anti-androgen RU58642. ${ }^{*} P<0.05$ and ${ }^{\dagger} P<0.001$ versus ORX control mice.

eration, whereas PSK3471, like tamoxifen or raloxifene (data not shown), did not display any agonist activity but rather inhibited $\mathrm{E}_{2}$-induced proliferation.

In conclusion, estrens act mostly as nonselective androgens that are also capable of acting via the ERs. Although they are capable of protecting from gonadectomy-induced bone loss in males and in females, their lack of selectivity induces unwanted responses in reproductive organs in vivo as well as in human breast cancer cells in vitro. Although these effects could be totally or in part mediated by nongenotropic signaling in respective target cells, estrens' ability to induce genotropic transcriptional activation at high concentrations has, in the absence of detailed pharmokinetic analysis here or in previous studies, made it impossible to draw any conclusions as to which mechanism is involved in the responses observed in vivo. Overall, these results are incompatible with the development of these molecules for the treatment or prevention of osteoporosis.

\section{Methods}

Animals. C57BL/6 mice were purchased from Charles River Laboratories. ER $\alpha^{-/-}$mice were generated and genotyped by PCR as described previously (26). ER $\alpha^{-/-}$mice were backcrossed for more than 6 genera- tions at the time of experiment and were therefore considered as being in pure C57BL/6 background.

Twelve-week-old male and female WT C57BL/6 mice were subjected to sham operation, ORX, or OVX. In order to establish the proper comparison with estren- $\alpha$, all compounds used in this study were delivered by slow-release pellets (Innovative Research of America). $\mathrm{E}_{2}$ was administered to female mice and DHT to male mice by slow-release pellets delivering $25 \mu \mathrm{g} / \mathrm{mouse} / 60$ days or $5 \mathrm{mg} /$ mouse/60 days, respectively. Both WT male and female mice were implanted with slow-release pellets delivering PSK3471 (50 $\mu \mathrm{g} / \mathrm{mouse} / 60$ days) or estren- $\alpha$ (7.6 mg/mouse/ 60 days). Both compounds were produced by ProStrakan. The properties of estren produced by ProStrakan were compared with those of estren obtained from Steraloids Inc., the supplier of estren used in the original studies (10). First, both Steraloids and ProStrakan estren behave exactly the same in in vitro assays. Second, the identity and purity of both preparations (Steraloids and ProStrakan) were checked by thin layer chromatography (TLC), NMR, and liquid chromatography/mass spectroscopy (LC/MS). TLC revealed that the ProStrakan estren compound was homogeneous (>98\% purity) and identical to the Steraloid reference batch. The NMR spectrum showed only signals due to the compound with signals of the ethanol used for the crystallization of the product. 
A

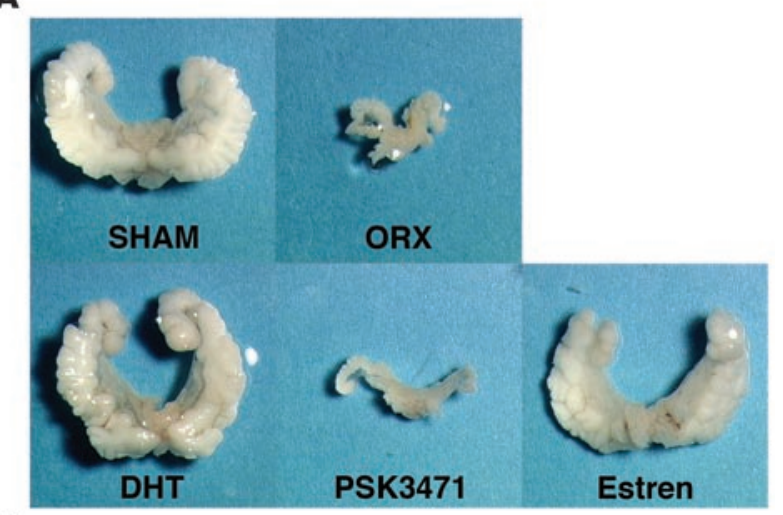

B

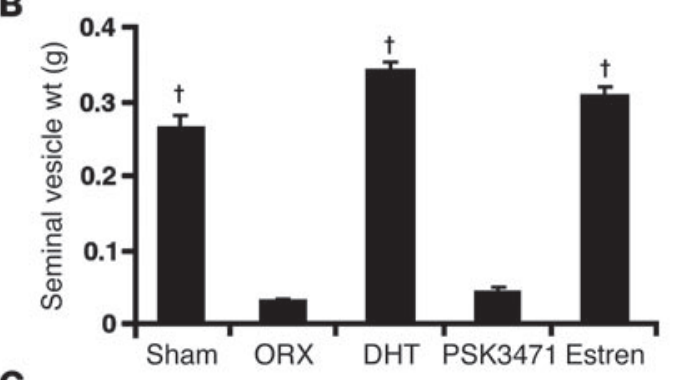

C

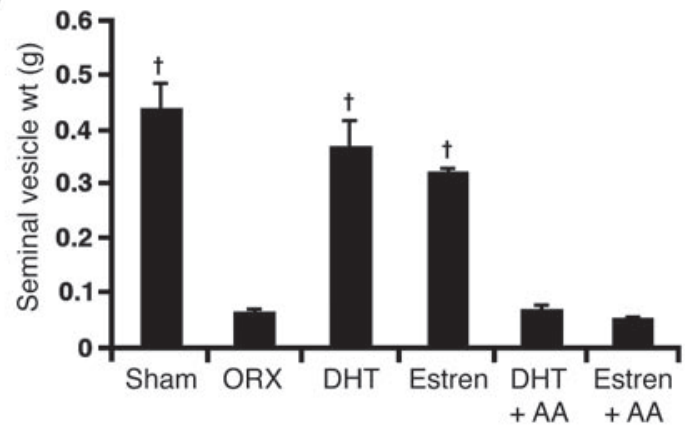

The ethanol content was estimated to be $12 \%$ by weight. The LC/MS spectrum was in accordance with the structure. Finally, the similarity of melting point of ProStrakan estren $\left(210^{\circ} \mathrm{C}\right)$ to that of Steraloid estren (206-207 ${ }^{\circ} \mathrm{C}$, according to the manufacturer) indicated that the crystalline structure of both compounds is the same. Anti-estrogen (PSK3668) and anti-androgen (RU58642) were administered at $10 \mathrm{mg} / \mathrm{kg} / \mathrm{d}$ and $30 \mathrm{mg} / \mathrm{kg} / \mathrm{d}$, respectively, by daily subcutaneous injection, as previously described (27). In the dose-response experiments, WT male C57BL/ 6 mice were subjected to ORX and treated with slow-release pellets delivering estren- $\alpha$ (Steraloids, $7.0 ; 1.8$ or $0.9 \mathrm{mg} / \mathrm{mouse} / 35$ days), DHT (Steraloids; $3.5 \mathrm{mg} /$ mouse $/ 35$ days), or vehicle. ER $\alpha^{-/-}$male mice were implanted with slow-release pellets delivering estren- $\alpha$ or estren- $\beta$ (Steraloids; $7 \mathrm{mg} /$ mouse/35 days), DHT, or vehicle. For all hormonal manipulations, treatment was begun at the time of operation and lasted for 4 weeks. Mice were housed in a standard animal facility under controlled temperature $\left(22^{\circ} \mathrm{C}\right.$ ) and photoperiod (12 hours of light, 12 hours of dark) with free access to water and standard food. All experiments in this study were approved by the Yale Animal Care and Use Committee or the ProStrakan Animal Care and Use Committee.

Peripheral quantitative CT. Densitometry was performed with the Stratec peripheral quantitative CT ( $\mathrm{PQCT}$ ) XCT Research SA + (software version 5.4B; Norland Medical Systems Inc.) operating at a resolution of $70 \mu \mathrm{m}$ as

\section{Figure 10}

Estren- $\alpha$ increases the weight of seminal vesicles in ORX WT and $\mathrm{ER} \alpha^{-/-}$ male mice. Male WT and $E R \alpha^{-1-}$ mice were subjected to sham operation or ORX and treatment with DHT, estren- $\alpha$, PSK3471, and/or the anti-androgen RU58642 as described in Methods. The seminal vesicles were weighed directly after the sacrifice. (A) Representative seminal vesicles from untreated and treated WT mice. (B) Quantification of the seminal vesicle wet weight in WT mice. (C) Quantification of the seminal vesicle wet weight in $\mathrm{ER} \alpha^{-1-}$ mice. ORX significantly reduced the seminal vesicle weight in both wild-type and $E R \alpha^{-/}$mice. Both estren- $\alpha$ and DHT induced seminal vesicle hypertrophy. These hypertrophic effects were abolished by coadministration of the anti-androgen RU58642 in ER $\alpha^{-1-}$ mice. ${ }^{\dagger} P<0.001$ versus matched ORX controls.

previously described (27). Briefly, metaphyseal PQCT scans of dissected right tibiae were performed to measure trabecular bone mineral density. The scan was positioned in the metaphysis at a distance from the distal growth plate corresponding to $6 \%$ of the total length of the tibia (an area containing cortical and trabecular bone). The trabecular bone region was defined by setting an inner area to $60 \%$ (peel 20 mode) of the total crosssectional area, as described previously (16). The interassay coefficients of variation for the PQCT measurements were less than $2.5 \%$ (16).

$X$-ray $\mu C T$ analysis of proximal tibiae. X-ray $\mu C T$ images were obtained on a Scanco Medical $\mu \mathrm{CT}$ scanner ( $\mu$ CT 20). The samples were stored in $70 \%$ ethanol for the duration of the scanning. A total of 223 images were obtained from each bone sample using a $512 \times 512$ matrix, resulting in an isotropic voxel resolution of $18 \times 18 \times 18 \mu \mathrm{m}^{3}$. An integration time of $100 \mathrm{~ms}$ per projection was utilized. Trabecular bone morphometric parameters were obtained from 2 sites $(1.0 \mathrm{~mm}$ and $2.5 \mathrm{~mm}$ from the growth plate) of the proximal tibiae of 10 -week-old mice, utilizing a spherical region of interest (volumes of $0.53-0.05 \mathrm{~mm}^{3}$ ) and built-in mean intercept length-based (MIL-based) analysis software. The raw images were gauss filtered $(\sigma=1.2$, base $=2)$ and binarized using a constant threshold before the morphometric analysis. The images were also rendered for 3D display and visualization. The measurements included bone volume density, bone surface density, trabecular number, trabecular thickness, and trabecular spacing.

Histomorphometry. Tibiae were collected at 4 weeks after operations (i.e., at 16 weeks of age), fixed in $3.7 \%$ formaldehyde in PBS, and embedded in methylmethacrylate (28). Double fluorochrome labeling was performed as described previously (28). Five-micrometer sections were stained with toluidine blue or analyzed unstained for fluorochrome labels according to standard procedures in the proximal tibia using the OsteoMeasure system (OsteoMetrics). Tibial cortical thickness and periosteal mineral appositional rates were measured as described previously (28).

Serum and urinary biochemistry. Serum osteocalcin and urinary Dpyr cross-links were evaluated with kits from Biomedical Technologies Inc. and Biometrics, respectively. Dpyr cross-link values were normalized to creatinine concentration to correct for water excretion. Serum $\mathrm{E}_{2}$ and testosterone were measured using human RIA kits adapted for mice (nos. 2464-07 and 2463-07; Immunotech).

Histology and immunohistochemistry. Uteri were weighed and then fixed in $4 \%$ formaldehyde overnight at $4^{\circ} \mathrm{C}$ and processed routinely for paraffin embedding. Five-micrometer-thick cross-sections were stained with Goldner's trichrome for histomorphometric analysis or with BrdU immunohistochemistry for assessing the proliferation index. For each mouse, cross-sections from both uterine horns were examined under a light microscope, and the following parameters were estimated: total uterus area $\left(\mathrm{mm}^{2}\right)$, endometrium thickness $(\mu \mathrm{m})$, and luminal epithelium height $(\mu \mathrm{m})$. 
Determination of relative binding affinities. The relative binding affinities of estrens and PSK3471 for ER $\alpha, E R \beta, A R$, glucocorticoid receptor (GR), PR, or mineralocorticoid receptor (MR) were determined by incubating crude cytosol from transfected COS-7 cells or baculovirus-infected Sf9 cells with the relevant $\left[{ }^{3} \mathrm{H}\right]$ ligand, with or without different concentrations of unlabeled competitor compound, for 24 hours at $0^{\circ} \mathrm{C}$. Bound and free ligands were separated by the dextran-coated charcoal method (29). The binding affinities of $\mathrm{E}_{2}(\mathrm{ERs})$, testosterone $(\mathrm{AR})$, dexamethasone $(\mathrm{GR})$, progesterone (PR), or aldosterone (MR) were taken as reference (100\%).

MCF-7 proliferation. MCF-7 cells were cultured in DMEM containing 5\% fetal calf serum (HyClone), sodium pyruvate (Invitrogen), nonessential amino acids (Invitrogen), and L-glutamine/penicillin/streptomycin solution (Sigma-Aldrich). The cells were grown for a week in the same medium with $50 \mathrm{ng} / \mathrm{ml}$ insulin and 5\% charcoal-stripped fetal calf serum and then detached and replated and cultured for an additional week in MEM-F12 ( $\mathrm{vol} / \mathrm{vol}$ ) without phenol red in the presence of $500 \mathrm{ng} / \mathrm{ml}$ insulin. Insulin was removed 2 days before the experiment. The cells $\left(6 \times 10^{4}\right.$ cells per well) were plated in 96-well plates, and the experiment was carried out in the above-mentioned medium. Twenty-four hours after seeding, the medium was changed, and growth was stimulated by $\mathrm{E}_{2}$ or the different test compounds dissolved in ethanol. The medium was changed 3 times a week. DNA content was evaluated on day 6 using HR33258 dye (30). The fluorescence (excitation $365 \mathrm{~nm}$, emission $460 \mathrm{~nm}$ ) was evaluated with a SPECTRAFluor Plus fluorimeter (TECAN).

Plasmids. The pC3-Luc vector was obtained from D. McDonnell (Duke University, Durham, North Carolina, USA). HEG0 (hER $\alpha$ expression vector) and PSG5AR (human AR expression vector) were obtained from P. Chambon (IGBMC, Illkirch, France). The plasmid pSG5-hER $\beta$ was constructed by inserting the entire coding sequence of the human ER $\beta$ at the BamHI site of the PSG5 vector. The PBARE-Luc reporter was constructed by inserting 3 copies of the ARE from the probasin promoter (31) upstream of the minimal promoter of collagenase (32) in the pGL3 vector (Promega) encoding for luciferase.

Transcriptional experiments. Stable ER $\alpha$ and ER $\beta$ reporter HeLa cell lines were obtained from P. Balaguer (INSERM U540, Montpellier, France) and have already been described (33). Cells were maintained in DMEM (Invitrogen) without phenol red, supplemented with $2 \mathrm{mM}$ L-glutamine, $1 \mathrm{mM}$ sodium pyruvate (Invitrogen), and nonessential amino acids (Invitrogen) and containing 10\% fetal calf serum (HyClone). The cells were maintained under pressure selection by adding puromycine $(0.25 \mu \mathrm{g} / \mathrm{ml})$ and G418 $(0.5 \mathrm{mg} / \mathrm{ml})$. To study hormonal effects, cells $\left(3 \times 10^{4}\right.$ cells per well) were seeded onto a 96-well culture plate in $100 \mu$ l of DMEM without phenol red and with $5 \%$ charcoal-stripped fetal calf serum. Twenty-four hours later, the medium was replaced, and hormones were added, as indicated in the figure legends, for 24 hours. Cells were then lysed in $100 \mu \mathrm{l}$ of lysis buffer (Tropix; Applied Biosystems), and luciferase activity was measured using the Luciferase Assay System (Promega). Triplicate determinations of reporter activity were performed in all experiments.
For transient transfections, HeLa cells were maintained in DMEM (Invitrogen) containing 5\% fetal calf serum (HyClone), sodium pyruvate (Invitrogen), nonessential amino acids (Invitrogen), and L-glutamine/ penicillin/streptomycin solution (Sigma-Aldrich). Cells were cultured in a humidified atmosphere containing $5 \% \mathrm{CO}_{2}$ at $37^{\circ} \mathrm{C}$.

For transfection experiments, HeLa cells $\left(6 \times 10^{4}\right.$ per well) were seeded in 24-well plates in DMEM without phenol red and containing 5\% charcoal/ dextran-stripped fetal calf serum and nonessential amino acids and L-glutamine. This medium was used throughout the experiment. Twenty-four hours later, cells were transfected with $25 \mathrm{ng}$ of CMV- $\beta$ Gal plasmid, $50 \mathrm{ng}$ of pC3-Luc vector, or $100 \mathrm{ng}$ of PBARE-Luc, together with $12.5 \mathrm{ng}$ of HEG0 or pSG5AR and $160 \mathrm{ng}$ of pBluescript plasmid (Stratagene) as a carrier, using the Lipofectamine PLUS reagent (Invitrogen) as recommended by the supplier. Eighteen hours later, the medium was changed for fresh medium containing the test compounds, and cells were incubated for a further 24 hours. Cells were then lysed in $300 \mu$ l of lysis buffer (Tropix; Applied Biosystems), and luciferase and $\beta$-galactosidase activities were measured using the Luciferase Assay System (Promega) and the Galacto Light kits (Tropix; Applied Biosystems), respectively. Luciferase activity was normalized to $\beta$-galactosidase activity to eliminate transfection efficiency differences in samples.

Statistics. All data are expressed as mean values \pm SEM and were statistically analyzed using nonparametric ANOVA followed by 2-tailed Student's $t$ test. $P$ values less than 0.05 were considered statistically significant. Each treated group was compared with the corresponding sham-operated and gonadectomized mice.

\section{Acknowledgments}

The work presented in this article was financially supported by a grant from ProStrakan Pharmaceuticals (to R. Baron) and fellowships from the Swedish Medical Research Council, the Swedish Foundation for International Cooperation in Research and Higher Education (STINT), the Foundation Blanceflor Boncompagni Ludovisi nee' Bildt, and the Helmut Hertz Foundation (to S.H. Windahl). We thank J.M. Lemoullec for providing the pBARE-Luc reporter plasmid.

Received for publication April 21, 2006, and accepted in revised form June 20, 2006.

Address correspondence to: Roland Baron, Department of Orthopaedics, Yale University School of Medicine, PO Box 208044, New Haven, Connecticut 06520-8044, USA. Phone: (203) 785-4150; Fax: (203) 785-2744; E-mail: roland.baron@yale.edu.

Sara H. Windahl's present address is: Center for Bone Research Department of Internal Medicine, Sahlgrenska Academy at Gothenburg University, Gothenburg, Sweden.

Sara H. Windahl and René Galien contributed equally to this work.
1. Rodan, G.A., and Martin, T.J. 2000. Therapeutic approaches to bone diseases. Science. 289:1508-1514.

2. McDonnell, D.P. 1999. The molecular pharmacology of SERMs. Trends Endocrinol. Metab. 10:301-311.

3. Riggs, B.L., and Hartmann, L.C. 2003. Selective estrogen-receptor modulators - mechanisms of action and application to clinical practice. N. Engl. J. Med. 348:618-629.

4. Lewis, J.S., and Jordan, V.C. 2005. Selective estrogen receptor modulators (SERMs): mechanisms of anticarcinogenesis and drug resistance. Mutat. Res. 591:247-263.

5. Love, R.R., et al. 1992. Effects of tamoxifen on bone mineral density in postmenopausal women with breast cancer. N. Engl. J. Med. 326:852-856.

6. [Anonymous]. 1998. Tamoxifen for early breast cancer: an overview of the randomised trials. Early Breast Cancer Trialists' Collaborative Group. Lancet. 351:1451-1467.

7. Fisher, B., et al. 1998. Tamoxifen for prevention of breast cancer: report of the National Surgical Adjuvant Breast and Bowel Project P-1 Study. J. Natl. Cancer Inst. 90:1371-1388.

8. Delmas, P.D., et al. 1997. Effects of raloxifene on bone mineral density, serum cholesterol concentrations, and uterine endometrium in postmenopausal women. N. Engl. J. Med. 337:1641-1647.

9. Manolagas, S.C. 2000. Birth and death of bone cells: basic regulatory mechanisms and implications for the pathogenesis and treatment of osteoporosis. Endocr. Rev. 21:115-137.

10. Kousteni, S., et al. 2002. Reversal of bone loss in mice by nongenotropic signaling of sex steroids. Science. 298:843-846.

11. Kousteni, S., et al. 2001. Nongenotropic, sex-nonspecific signaling through the estrogen or androgen receptors: dissociation from transcriptional activity. Cell. 104:719-730.

12. Manolagas, S.C., Kousteni, S., and Jilka, R.L. 2002. Sex steroids and bone. Recent Prog. Horm. Res. 57:385-409.

13. Moggs, J.G., Deavall, D.G., and Orphanides, G. 
2003. Sex steroids, ANGELS and osteoporosis. Bioessays. 25:195-199.

14. Centrella, M., McCarthy, T.L., Chang, W.Z., Labaree, D.C., and Hochberg, R.B. 2004. Estren (4estren-3alpha,17beta-diol) is a prohormone that regulates both androgenic and estrogenic transcriptional effects through the androgen receptor. Mol. Endocrinol. 18:1120-1130.

15. Krishnan, V., et al. 2005. The nongenotropic synthetic ligand 4-estren-3alpha17beta-diol is a highaffinity genotropic androgen receptor agonist. Mol. Pharmacol. 67:744-748.

16. Sims, N.A., et al. 2002. Deletion of estrogen receptors reveals a regulatory role for estrogen receptors-beta in bone remodeling in females but not in males. Bone. 30:18-25.

17. Rossouw, J.E., et al. 2002. Risks and benefits of estrogen plus progestin in healthy postmenopausal women: principal results from the Women's Health Initiative randomized controlled trial. JAMA. 288:321-333.

18. Shumaker, S.A., et al. 2003. Estrogen plus progestin and the incidence of dementia and mild cognitive impairment in postmenopausal women: the Women's Health Initiative Memory Study; a randomized controlled trial. JAMA. 289:2651-2662.

19. Beral, V. 2003. Breast cancer and hormone-replacement therapy in the Million Women Study. Lancet.
362:419-427

20. Bhasin, S., et al. 1998. Issues in testosterone replacement in older men. J. Clin. Endocrinol. Metab. 83:3435-3448.

21. Buijsman, R.C., Hermkens, P.H., van Rijn, R.D., Stock, H.T., and Teerhuis, N.M. 2005. Non-steroidal steroid receptor modulators. Curr. Med. Chem. 12:1017-1075.

22. Qoubaitary, A., Swerdloff, R.S., and Wang, C. 2005. Advances in male hormone substitution therapy. Expert Opin. Pharmacother. 6:1493-1506.

23. Moverare, S., et al. 2003. Estren is a selective estrogen receptor modulator with transcriptional activity. Mol. Pharmacol. 64:1428-1433.

24. Wessler, S., Otto, C., Wilck, N., Stangl, V., and Fritzemeier, K.H. 2006. Identification of estrogen receptor ligands leading to activation of nongenomic signaling pathways while exhibiting only weak transcriptional activity. J. Steroid Biochem. Mol. Biol. 98:25-35.

25. Hewitt, S.C., Collins, J., Grissom, S., Hamilton, K., and Korach, K.S. 2006. Estren behaves as a weak estrogen rather than a non-genomic selective activator in the mouse uterus. Endocrinology. 147:2203-2214.

26. Dupont, S., et al. 2000. Effect of single and compound knockouts of estrogen receptors alpha (ERalpha) and beta (ERbeta) on mouse reproductive phenotypes. Development. 127:4277-4291.
27. Sims, N.A., et al. 2003. A functional androgen receptor is not sufficient to allow estradiol to protect bone after gonadectomy in estradiol receptor-deficient mice. J. Clin. Invest. 111:1319-1327. doi:10.1172/JCI200317246.

28. Sims, N.A., et al. 2000. Bone homeostasis in growth hormone receptor-null mice is restored by IGF-I but independent of Stat5. J. Clin. Invest. 106:1095-1103.

29. Vayssiere, B.M., et al. 1997. Synthetic glucocorticoids that dissociate transactivation and AP-1 transrepression exhibit antiinflammatory activity in vivo. Mol. Endocrinol. 11:1245-1255.

30. Rao, J., and Otto, W.R. 1992. Fluorimetric DNA assay for cell growth estimation. Anal. Biochem. 207:186-192.

31. Claessens, F., et al. 1996. The androgen-specific probasin response element 2 interacts differentially with androgen and glucocorticoid receptors. J. Biol. Chem. 271:19013-19016.

32. Angel, P., et al. 1987. 12-O-tetradecanoyl-phorbol13-acetate induction of the human collagenase gene is mediated by an inducible enhancer element located in the $5^{\prime}$-flanking region. Mol. Cell. Biol. 7:2256-2266.

33. Balaguer, P., Boussioux, A.M., Demirpence, E., and Nicolas, J.C. 2001. Reporter cell lines are useful tools for monitoring biological activity of nuclear receptor ligands. Luminescence. 16:153-158. 